\title{
Theoretical constraints for the magnetic-dimer transition in two-dimensional spin models
}

\author{
Leonardo Spanu, ${ }^{1}$ Federico Becca, ${ }^{1,2}$ and Sandro Sorella ${ }^{1}$ \\ ${ }^{1}$ INFM-Democritos, National Simulation Center and International School for Advanced Studies (SISSA), I-34014 Trieste, Italy \\ ${ }^{2}$ Laboratoire de Physique Théorique, Université "Paul Sabatier", F-31062 Toulouse, France
}

(Dated: June 26, 2018)

\begin{abstract}
From general arguments, that are valid for spin models with sufficiently short-range interactions, we derive strong constraints on the excitation spectrum across a continuous phase transition at zero temperature between a magnetic and a dimerized phase, that breaks the translational symmetry. From the different symmetries of the two phases, it is possible to predict, at the quantum critical point, a branch of gapless excitations, not described by standard semi-classical approaches. By using these arguments, supported by intensive numerical calculations, we obtain a rather convincing evidence in favor of a first-order transition from the ferromagnetic to the dimerized phase in the two-dimensional spin-half model with four-spin ring-exchange interaction, recently introduced by A.W. Sandvik et al. [Phys. Rev. Lett. 89, 247201 (2002)].
\end{abstract}

PACS numbers:

\section{INTRODUCTION}

In the last years, a large amount of work has been done to clarify the properties of unconventional magnetic systems. Indeed, the presence of frustrated interactions, generated either by the geometry of the lattice or by the competing interactions, can give rise to many anomalous low-energy properties. $\frac{1}{-}$ One of the most exciting is the possibility to obtain phases with no magnetic order even at zero temperature ${ }^{2}$ The actual interest in this subject has been recently renewed by the discovery of several materials 3.4 .5 that do not show any sign of magnetic order down to very low temperatures. From a theoretical point of view, a considerable progress has been done in elucidating the possible unconventional states and, apart from magnetic phases, it is now well accepted that many systems exhibit a large variety of disordered ground states. One class of such paramagnetic states is given by the so-called valence-bond solids, where pairs of nearest-neighbor spins form a singlet, leading to an ordered pattern of valence bonds. These states explicitly break the translational symmetry, implying a degenerate ground state, and represent the two-dimensional extension of the celebrated dimerized state, rather well established in quasi-one-dimensional materials ${ }^{6}$ Another class of disordered phases is given by the so-called spin liquids, that do not break any local symmetry. It is now rather widely accepted that also spin liquids possess some degeneracy, or even a gapless spectrum, and have some kind of order, called topological order, related to non-local operators 7,8

Much recent research has focused on the way to describe transitions between different quantum phases. Indeed, from the Landau's arguments valid for classical critical phenomena, if one exclude a delicate fine tuning of the parameters, there is no reason to have a continuous transition between two phases with different symmetries of the order parameters, like for instance between a mag- netic system and a valence-bond solid, and a first-order transition would be the most natural scenario. However, very recently, 9.10 these arguments have been questioned and it has been argued that the situation can be more complex and richer in quantum systems, where the Landau paradigm could be violated, giving rise to an appealing way to escape a first-order transition. Therefore, it has been suggested that a quantum phase transition is intrinsically different from a classical one. In particular, when considering quantum phases, the natural description is no longer by using order parameters but instead by considering new degrees of freedom carrying fractional quantum numbers, that emerge at the critical point.

The arguments given in Refs. 9 and 10 , based on fieldtheory approaches, are rather convincing but it is natural to ask if microscopic spin models, defined on a lattice, could present the same low-energy critical behavior. In this sense, however, we face a technical difficulty: a good diagnosis would require to study large enough clusters, but this is not possible since quantum Monte Carlo simulations of frustrated antiferromagnets are plagued with a very severe minus sign problem. In this respect, it is important to find out microscopic models that do not suffer from this problem but, at the same time, present phase transitions between quantum phases. Recently, Sandvik and collaborators ${ }^{11}$ introduced a spin-half model on the two-dimensional square lattice in the presence of a particular ring-exchange interaction:

$$
\mathcal{H}=\mathcal{H}_{\mathrm{XY}}+\mathcal{H}_{\text {Ring }},
$$

where

$$
\begin{aligned}
\mathcal{H}_{\mathrm{XY}} & =-\frac{J}{2} \sum_{\langle i, j\rangle}\left(S_{i}^{+} S_{j}^{-}+S_{i}^{-} S_{j}^{+}\right) \\
\mathcal{H}_{\mathrm{Ring}} & =-K \sum_{[i, j, k, l]}\left(S_{i}^{+} S_{j}^{-} S_{k}^{+} S_{l}^{-}+S_{i}^{-} S_{j}^{+} S_{k}^{-} S_{l}^{+}\right) .
\end{aligned}
$$

Here $\langle i, j\rangle$ denotes a pair of nearest neighbor sites and $[i, j, k, l]$ indicates the sites on the corners of a plaque- 
tte. In particular, $\mathcal{H}_{\text {Ring }}$ is a ring-exchange interactions that acts as a cyclic permutation on the spins of a given plaquette whenever they are in a $S^{z}=0$ configuration with parallel spins on opposite corners ${ }^{12}$ The case with $J>0$ and $K>0$ is considered, in order to avoid the sign problem. Notice that $\mathcal{H}$ is invariant under global U(1) transformations $S_{i}^{+} \rightarrow e^{i \theta} S_{i}^{+}$.

The Hamiltonian (1) has been investigated by using quantum Monte Carlo ${ }^{11,13}$ and it has been shown that, at zero temperature and zero magnetic field, there is a transition between a ferromagnetic phase, stable for small values of $K / J$, and a dimerized one, characterized by columnar order at $Q=(\pi, 0)$ and $Q=(0, \pi)$, stable for intermediate ring-exchange couplings. Finally, for large $K / J$ a checkerboard phase is also expected. ${ }^{11.13}$ Note that, in our notation, $J$ is twice time bigger than the one used in Refs. 11 and 13 . Once scaled in our units, their best estimate for the transition point from the magnetic to the dimerized phase is $K_{c} / J \sim 3.957 .14$ In the original paper, ${ }^{11}$ it has been shown that, by increasing the strength of the ring exchange, both the spin stiffness $\rho_{s}$ and the magnetization $M$ decrease and eventually vanish, with a corresponding insurgence of a finite dimer order parameter. Hence, the Hamiltonian (11) has been suggested as a prototype model to test the ideas of Refs. 9 and 10 . However, subsequent and more accurate calculations have raised some doubt on the previous ones, suggesting instead a weak first-order transition. 15 The actual nature of the transition is therefore still controversial.

In this paper, we propose a different point of view to investigate the properties of a phase transition between a magnetic and a dimerized phase of a generic spin Hamiltonian with sufficiently short-range interactions. Indeed, instead of calculating the order parameters and the stiffness, we derive strong constraints on the excitation spectrum across a continuous phase transition between a magnetically ordered and a dimerized phase, breaking translational symmetry. These constraints can be verified by using numerical techniques, such as the Green's function Monte Carlo method. ${ }^{16}$ In our opinion this way to proceed represents the first attempt to clarify the evolution of the excitation spectrum and can give important insight into the nature of the transition point also in other contexts.

The paper is organized as follow: in Sec. III we develop the general theoretical formalism, in Sec. III we present our numerical results, and in Sec. IV] we draw our conclusions.

\section{THEORETICAL FORMALISM}

In this section, we describe in detail the implications of a continuous phase transition from a magnetically ordered phase to a dimerized one on the excitation spectrum near the quantum critical point. In the following, we will consider an Hamiltonian with a generic transla- tional invariant two-spin coupling and $\mathrm{SU}(2)$ symmetry, and at the end of the section we will also discuss the cases with $\mathrm{U}(1)$ symmetry or multi-spin interactions.

Let us consider the Heisenberg Hamiltonian on a twodimensional lattice with $N=L \times L$ sites:

$$
\mathcal{H}=\sum_{i, j} J_{i, j} \vec{S}_{i} \cdot \vec{S}_{j}
$$

where $J_{i, j}$ is the coupling between the spins at site $R_{i}$ and $R_{j}$ and $\vec{S}_{i}=\left(S_{i}^{x}, S_{i}^{y}, S_{i}^{z}\right)$ is the spin operator at the site $R_{i}$. For a translational invariant magnetic coupling that depends only upon $\left|R_{i}-R_{j}\right|$, i.e., $J_{i, j}=J_{|i-j|}=J_{r}$, we can perform the Fourier transform and easily obtain:

$$
\mathcal{H}=\sum_{q} J_{q} \vec{S}_{q} \cdot \vec{S}_{-q}
$$

where $\vec{S}_{q}=1 / \sqrt{N} \sum_{j} e^{i q R_{j}} \vec{S}_{j}$ and $J_{q}=\sum_{r} e^{i q R_{r}} J_{r}$ define the Fourier transform of the local spin operator and the magnetic coupling, respectively. In the following, we will consider interactions that are sufficiently short range, that is:

$$
\tilde{J}=\frac{1}{2 N} \sum_{i, j}\left|J_{i, j}\right|\left|R_{i}-R_{j}\right|^{2}<\infty
$$

For the $\mathrm{SU}(2)$ case, the eigenstates can be classified according to the total spin $S$ and the ground state, here denoted by $\left|\Psi_{Q_{0}}\right\rangle$, is generally a singlet with momentum $Q_{0}$. By means of the so-called Feynman construction, originally introduced to describe the excitations of the liquid Helium, ${ }^{17}$ we can easily define a variational state for the triplet excited state with momentum $q$ with respect to the ground state:

$$
\left|\Psi_{Q_{0}+q}\right\rangle=S_{q}^{\alpha}\left|\Psi_{Q_{0}}\right\rangle
$$

$S_{q}^{\alpha}$ being one of the three components of the total spin $\vec{S}_{q}$ $(\alpha=x, y, z)$. Then, it is easy to calculate the variational energy $E_{Q_{0}+q}$ of $\left|\Psi_{Q_{0}+q}\right\rangle$

$$
\Delta\left(Q_{0}+q\right)=E_{Q_{0}+q}-E_{Q_{0}}=\frac{F_{q}^{\alpha}}{\left\langle S_{-q}^{\alpha} S_{q}^{\alpha}\right\rangle},
$$

where $\langle\ldots\rangle$ stands for the average value over $\left|\Psi_{Q_{0}}\right\rangle$ and the function $F_{q}^{\alpha}$ is given by the double commutator

$$
F_{q}^{\alpha}=\frac{1}{2}\left\langle\left[S_{-q}^{\alpha},\left[\mathcal{H}, S_{q}^{\alpha}\right]\right]\right\rangle .
$$

By using standard commutation relations for the spin operators and by summing over all the spin components $\alpha$, we obtain

$$
\sum_{\alpha} F_{q}^{\alpha}=\frac{1}{2 N} \sum_{k}\left[J_{k+q}+J_{k-q}-2 J_{k}\right]\left\langle\vec{S}_{k} \cdot \vec{S}_{-k}\right\rangle .
$$


After performing the inverse Fourier transform, we have

$$
\begin{aligned}
& \sum_{\alpha} F_{q}^{\alpha}=\frac{1}{N} \sum_{i, j} J_{i, j}\left\{\cos \left[q\left(R_{i}-R_{j}\right)\right]-1\right\}\left\langle\vec{S}_{i} \cdot \vec{S}_{j}\right\rangle \leq \\
& \frac{q^{2}}{2 N} \sum_{i, j}\left|J_{i, j}\right|\left|R_{i}-R_{j}\right|^{2}\left|\left\langle\vec{S}_{i} \cdot \vec{S}_{j}\right\rangle\right| \leq \tilde{J} S(S+1) q^{2},
\end{aligned}
$$

where we used that $\left|\left\langle\vec{S}_{i} \cdot \vec{S}_{j}\right\rangle\right| \leq S(S+1)$ and the shortrange condition (4). Notice that the previous bound does not rely upon a small- $q$ expansion, but it is instead valid for all momenta. Therefore, we arrive to the important result that:

$$
\Delta\left(Q_{0}+q\right) \leq \frac{\tilde{J} S(S+1) q^{2}}{S(q)},
$$

where $S(q)=\left\langle S_{-q}^{\alpha} S_{q}^{\alpha}\right\rangle$ is the static magnetic structure factor, that, in the $\mathrm{SU}(2)$ case, does not depend upon $\alpha$. Since $\left|\Psi_{Q_{0}+q}\right\rangle$ is a variational state, its average energy has to be higher than the lowest exact triplet excitation with given momentum $Q_{0}+q$. We conclude, therefore, that the inequality (10) will also hold for the exact triplet excitation energy with momentum $Q_{0}+q$.

In the following we will argue that the rigorous bound on the excitation spectrum (10) quite naturally implies the existence of a new branch of gapless excitations close to an hypothetical second-order phase transition from a magnetic phase to a dimerized one. We will focus on the square lattice case and an ordered phase with staggered magnetization at $Q=(\pi, \pi)$, but similar results can be also found in the case of different lattice structures or spiral magnetic orders. For the sake of simplicity, we can imagine that $J_{q}$ depends upon one parameter $g$, proportional to the frustration of model: for instance, in the $J_{1}-J_{2}$ model $J_{q}=2\left(\cos q_{x}+\cos q_{y}\right)+4 g \cos q_{x} \cos q_{y}$, with $g=J_{2} / J_{1}$. Suppose that, by increasing the frustrating parameter $g$, the system has a continuous transition from a magnetically ordered phase to a dimerized one. In this latter, the lowest triplet excitation remains gapped in the thermodynamic limit and the ground state is fourfold degenerate, with four different momenta $q=(0,0)$, $(0, \pi),(\pi, 0)$, and $(\pi, \pi)$. Again, the following arguments will also hold for any other dimer patterns, having ground states with momenta $q \neq(0,0)$ and $q \neq(\pi, \pi)$. By applying the Feynman construction with $q=(\pi, \pi)$ to the state with $q=(0, \pi)$ and by using the inequality (10), we obtain that

$$
\Delta(\pi, 0) \leq \frac{2 \pi^{2} \tilde{J} S(S+1)}{S(\pi, \pi)} .
$$

In the dimerized region, i.e., for $g>g_{c}$, the spectrum is gapped, implying that $S(\pi, \pi)$ is finite, and the constraint (11) does not give us any useful information on the excitations. However, if the transition is continuous, the four-fold degeneracy will hold down to the critical point $g=g_{c}$, where $S(\pi, \pi)$ diverges, because of the incipient magnetic phase, stable for $g<g_{c}$. Indeed, at the critical
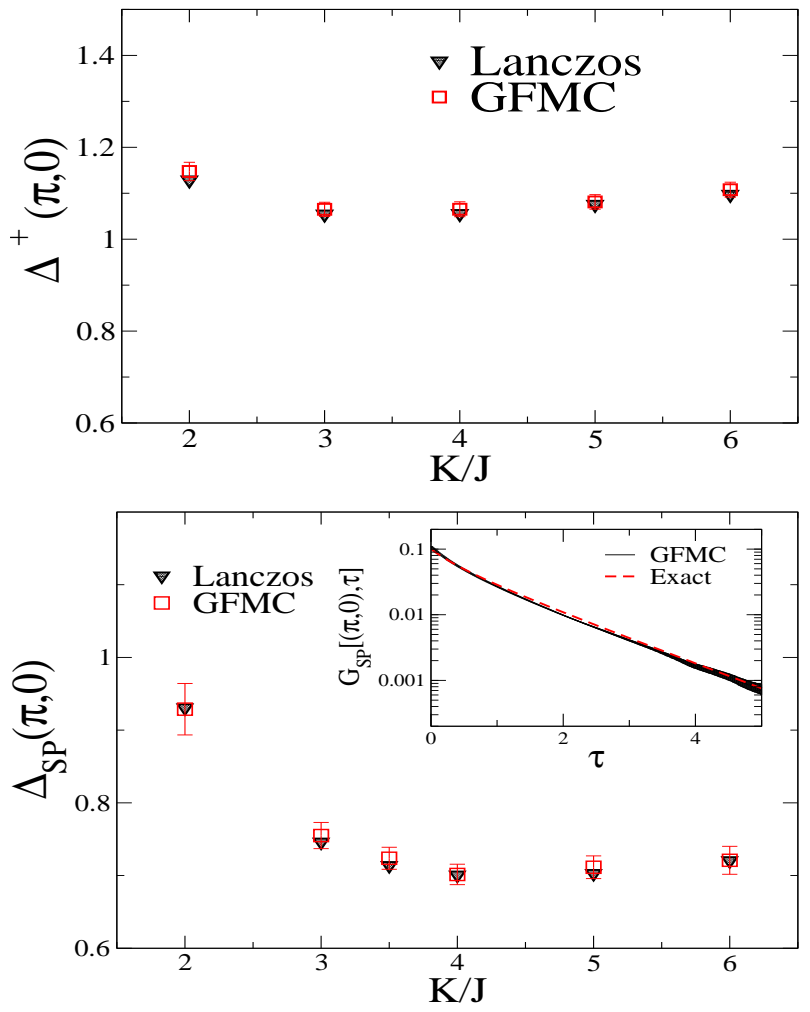

FIG. 1: (Color online) Comparison on a $6 \times 6$ lattice between Lanczos and GFMC for the lowest energy gap. Upper panel: $\Delta^{+}(\pi, 0)$ (see text) [in unit of $(K+J)$ ], as a function of $K / J$. Lower panel: $\Delta_{S P}(\pi, 0)$ (see text) [in unit of $(K+J)$ ], as a function of $K / J$. Inset: direct comparison of the dynamical correlation function for the dimer operator calculated by GFMC approach and by Lanczos.

point the spin-spin correlations are expected to decay as $|R|^{-(1+\eta)}$, leading to $S(\pi, \pi) \sim L^{1-\eta}$. The actual value of $\eta$ can be found by considering the corresponding classical model in three dimensions. In the Heisenberg model with nearest-neighbor interactions, we have that $\eta \sim 0$, implying a rather strong divergence of the static structure factor at the critical point. However, this divergence can be much weaker, i.e., $\eta \sim 0.5$, in the case where no free topological singularities are allowed but only pairs of them are present ${ }^{18,19}$ We point out here that our conclusions remain valid as long as $\eta \leq 1$, the structure factor being logarithmically divergent in the case of $\eta=1$.

Therefore, because the operator $S_{q}^{\alpha}$ used in the Feynman construction carries $S=1$, we immediately arrive to the conclusion that Eq. (11) implies the existence of a branch of excitations, with $S=1$ and $q=(\pi, 0)$, that becomes gapless at $g=g_{c}$. Analogously, by considering the ground state with momentum $q=(\pi, 0)$, we obtain a branch of excitations with $S=1$ and $q=(0, \pi)$. This outcome contrasts the common understanding of the lowenergy properties of magnetic systems with $\mathrm{O}(3)$ symmetry, which contains only two branches of gapless excitations at $q=(0,0)$ and $q=(\pi, \pi)$, while the triplet excitations at $q=(0, \pi)$ and $q=(0, \pi)$ are always gapped ${ }^{20}$ 


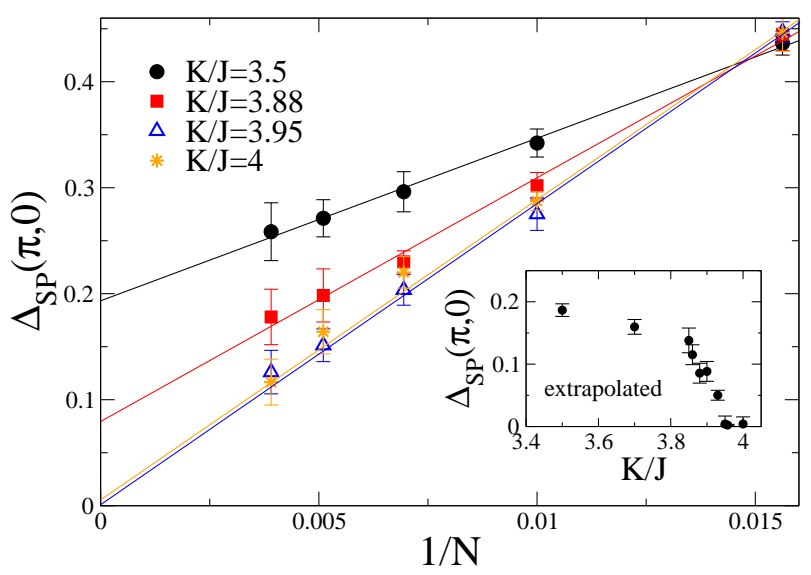

FIG. 2: (Color online) The dimer gap $\Delta_{S P}(\pi, 0)$ [in unit of $(K+J)$ ] obtained by GFMC as a function of the size $N$ of the cluster for different values of $K / J$. The lines are linear fits of the points. Inset: the extrapolated value in the thermodynamic limit as a function of $K / J$.

Therefore, one is left with the following three possibilities: the first one is to have an $\mathrm{O}(3)$ critical point with more than two gapless modes, not described by standard theories of magnetic phase transitions. The second one is that the dimerized phase is separated from the magnetic phase by a first-order transition: in that case the spectrum can evolve discontinuously and the triplet states at $q=(0, \pi)$ and $q=(0, \pi)$ can remain gapped. The third possibility is that there is still a continuous transition but the non-magnetic phase does not break the translational invariance and, therefore, has no dimer order. Then, the dimerized phase could be eventually stabilized through a further (continuous) transition.

We stress that a continuous transition between a magnetically ordered phase and a dimerized one is instead possible in models that explicitly break the translational invariance, like for instance the coupled ladder system, 21 where due to the folding of the Brillouin zone $q=(\pi, 0)$ [or $q=(0, \pi)]$ is equivalent to $q=(0,0)$.

Now we would like to discuss what happens for systems with different kinds of interactions. In the case of multispin interactions, it is easy to prove that we can arrive to similar expressions and the only difference is the prefactor, due to a different value of the double commutator (7). A little more care must be paid to a model with U(1) symmetry. Here, the total spin $S$ does not commute with the Hamiltonian, and only $S^{z}$ is a good quantum number. Furthermore, the states with $S^{z}=0$ can be also classified according the discrete particle-hole symmetry $(\mathrm{PH})$ $S^{z} \rightarrow-S^{z}$. In the weakly frustrated limit, without loss of generality, we can consider the ferromagnetic $X Y$ model, that has only one gapless branch at $q=(0,0)$. On a finite cluster, the ground state has $S^{z}=0$ and can be taken to be even under the PH symmetry, i.e., with $P H=+1$. In this case, we can construct two different variational states for the low-energy excitations. The first one, with $S^{z}=1$, is given by $\left|\Psi_{Q_{0}+q}\right\rangle=S_{q}^{+}\left|\Psi_{Q_{0}}\right\rangle, S_{q}^{+}$being the
Fourier transform of $S_{j}^{+}=S_{j}^{x}+i S_{j}^{y}$, and has

$$
\Delta^{+}\left(Q_{0}+q\right)=\frac{F_{q}^{+}}{\left\langle S_{-q}^{-} S_{q}^{+}\right\rangle} .
$$

Instead, the second possibility, with $S^{z}=0$ and $P H=$ -1 , is given by $\left|\Psi_{Q_{0}+q}\right\rangle=S_{q}^{z}\left|\Psi_{Q_{0}}\right\rangle$ and has

$$
\Delta^{z}\left(Q_{0}+q\right)=\frac{F_{q}^{z}}{\left\langle S_{-q}^{z} S_{q}^{z}\right\rangle} .
$$

In the dimerized phase the ground state is four-fold degenerate with four different momenta $q=(0,0),(0, \pi)$, $(\pi, 0)$, and $(\pi, \pi)$, all of them having $S^{z}=0$ and $P H=$ +1 this is equivalent to have all singlets in the $\mathrm{SU}(2)$ case]. Then, in close relation with the $\mathrm{SU}(2)$ case, the Feynman construction (12) with $q \rightarrow(0,0)$ applied to the state with momentum $q=(\pi, 0)$ implies the existence of a gapless branch of excitations at $q=(\pi, 0)$ with $S^{z} \neq 0$. Indeed, in this case $F_{q}^{+}$is finite for $|q| \rightarrow 0$ and the structure factor in the denominator diverges at the critical point, as $\left\langle S_{-q}^{-} S_{q}^{+}\right\rangle \propto 1 / q^{1-\eta}$. Hence, we arrive to the same conclusions as before: excluding the possibility of having more than one gapless branch in a model with $\mathrm{O}(2)$ symmetry, in contrast with semiclassical approaches 20 the transition must be either first order or second order to a non-dimerized phase. Finally, it should be noted that, under an additional assumption, also Eq. (13) gives a strict bound to the spectrum. Indeed, $F_{q}^{z} \propto q^{2}$ for $|q| \rightarrow 0$ and, whenever at the critical point $\left\langle S_{-q}^{z} S_{q}^{z}\right\rangle$ does not vanish too fast, i.e., $\left\langle S_{-q}^{z} S_{q}^{z}\right\rangle \propto q^{2-\alpha}$ with $\alpha>0$, also $\Delta^{z}(q) \rightarrow 0$ for $|q| \rightarrow 0$.

\section{NUMERICAL RESULTS}

In this section, we present our numerical results for the Hamiltonian (11), by using the Green's function Monte Carlo (GFMC) technique with a finite and fixed population of walkers $\stackrel{16}{1 n}$ order to minimize the statistical fluctuations, we implemented the importance sampling, defined by the variational wave function: 22

$$
\left|\Phi_{V}\right\rangle=\mathcal{P}_{z} \mathcal{J}|F\rangle
$$

where $|F\rangle$ is the ferromagnetic state with the magnetization along the $x$ direction, i.e., $|F\rangle=\Pi_{i}\left(|\uparrow\rangle_{i}+|\downarrow\rangle_{i}\right), \mathcal{P}_{z}$ is the projector onto the subspace with $S^{z}=0$, and $\mathcal{J}$ is a spin Jastrow factor

$$
\mathcal{J}=\exp \left\{\frac{1}{2} \sum_{i, j} v_{i, j} S_{i}^{z} S_{j}^{z}\right\},
$$

with the two-body potential $v_{i, j}$ that depends only upon the distance $\left|R_{i}-R_{j}\right|$ and can be optimized by using the minimization technique described in Ref. 23.

Since the Hamiltonian (1) does not suffer from the sign problem, it is possible to sample the exact ground-state 


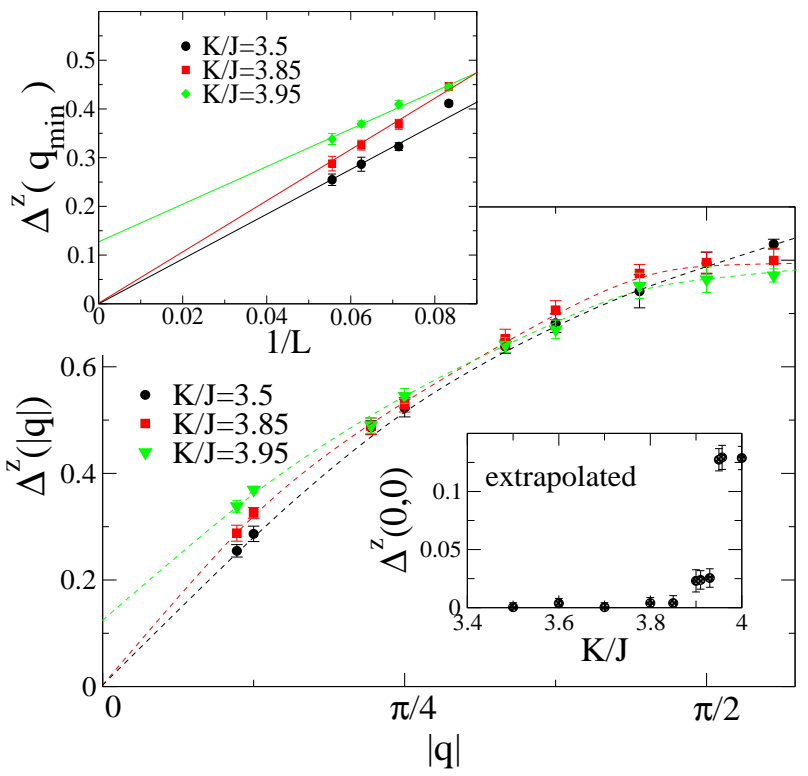

FIG. 3: (Color online) The spin-wave spectrum [in unit of $(K+J)$ ] obtained by GFMC by using the operator $O_{q}=S_{q}^{z}$. The linear sizes of the cluster are $L=16$ and 18, and the lines are guides to the eye. The vector $q$ is taken along the $(1,0)$ direction. Upper inset: the behavior of the finite-size gap at the minimum momentum, i.e., $q_{\min }=(2 \pi / L, 0)$ as a function of $L$ for different values of $K / J$. Lower inset: the thermodynamic limit of the gap at $q=(0,0)$ as a function of $K / J$.

wave function $\Phi_{0}(x)=\left\langle x \mid \Phi_{0}\right\rangle$ (being $|x\rangle$ a generic spin configuration), or, with importance sampling, the positive quantity $\Phi_{0}(x) \Phi_{V}(x)=\left\langle x \mid \Phi_{0}\right\rangle\left\langle x \mid \Phi_{V}\right\rangle$. Moreover, it is possible to evaluate the imaginary time evolution of the following dynamical correlation function:

$$
G(q, \tau)=\frac{\left\langle\Phi_{V}\left|O_{-q} e^{-\tau \mathcal{H}} O_{q}\right| \Phi_{0}\right\rangle}{\left\langle\Phi_{V}\left|e^{-\tau \mathcal{H}}\right| \Phi_{0}\right\rangle}
$$

where $O_{q}$ is a given operator with a definite momentum $q$. This technique has been already successfully used by one of us to compute the excitation spectrum of a quasione-dimensional magnetic system ${ }^{24}$ It is easy to show that, for large imaginary time $\tau, G(q, \tau)$ behaves like:

$$
G(q, \tau) \propto e^{-\tau \Delta_{q}},
$$

where $\Delta_{q}=E_{q}-E_{0}$ is the energy gap between the ground state and the first excited state $\left|\Phi_{q}\right\rangle$ such that

$$
\left\langle\Phi_{q}\left|O_{q}\right| \Phi_{0}\right\rangle \neq 0 .
$$

Therefore, from the fit of the large- $\tau$ behavior of the dynamical correlation function, it is possible to extract the lowest gap with a given momentum $q$ and the symmetry properties imposed by the condition (18).

Whenever the operator $O_{q}$ is diagonal in the spin configuration $|x\rangle$, like, for instance the case of $S_{q}^{z}$, the expression (16) can be easily calculated by using the standard forward walking technique. ${ }^{16}$ On the other hand, for non-diagonal operators, i.e., for $S_{q}^{+}$, a more involved calculation is needed, since the operator breaks the Markov chain of the Monte Carlo simulation. In order to avoid this extra numerical effort, we have calculated more efficiently $\Delta^{+}(q)$ by sampling the ground state with $S^{z}=1$ and by calculating its excitation spectrum, for instance by using $S_{q}^{z}$. Then, $\Delta^{+}(q)$ can be found from:

$$
\begin{aligned}
\Delta^{+}(q) & =E_{q}^{S^{z}=1}-E_{0}^{S^{z}=0} \\
& =\left(E_{q}^{S^{z}=1}-E_{0}^{S^{z}=1}\right)+E_{0}^{S^{z}=1}-E_{0}^{S^{z}=0},
\end{aligned}
$$

where the term in the first bracket can be calculated by fitting $G(q, \tau)$ and the last two terms are just the groundstate energies of the two sectors with $S^{z}=0$ and $S^{z}=1$, easily calculated by GFMC.

In order to verify the accuracy of GFMC for the evaluation of the gap, we can perform calculations on a $6 \times 6$ lattice, where the exact results are available by the Lanczos method. In particular, we consider the dimer operator $O_{q}=O_{q}^{S P}=1 / \sqrt{N} \sum_{j} e^{i q R_{j}} S_{j}^{z} S_{j+x}^{z}$ with $q=(\pi, 0)$. In this case, the operator $O_{q}$ does not change the $P H$ quantum number and we have access to the lowest gap with the same $P H$ of the ground state. In Fig. 1 we compare, for different values of the ring-exchange coupling $K / J$, the exact energy gap at $q=(\pi, 0)$ and even under $P H$ with the one extracted from the large- $\tau$ behavior of $G(q, \tau)$, and denoted by $\Delta_{S P}(\pi, 0)$. Moreover, as a further check of the statistical approach, we can also compute exactly the dynamical correlation function and make the comparison with the GFMC calculations (see the inset of Fig. 10. It should be stressed that, for our purpose, we are only interested in the lowest energy gap, and, therefore, we fit only the large- $\tau$ part of $G(q, \tau)$. This can be achieved by using only two or three exponents at most ${ }^{25}$ In the same figure, we also report the comparison for $\Delta^{+}(\pi, 0)$, extracted from the decomposition of Eq. (19): also in this case the agreement is excellent.

The lowest dimer gap $\Delta_{S P}(\pi, 0)$ provides us the informations on the appearance of a dimerized phase with spin-Peierls order. In Fig. 2 we report the size-scaling calculations of $\Delta_{S P}(\pi, 0)$ as a function of the inverse of the size $N$, for different values of the ring exchange. The dimer gap is clearly finite in the magnetically ordered phase, with a decreasing behavior by approaching the transition. The thermodynamic limit indicates a vanishing value of $\Delta_{S P}(\pi, 0)$ for $K / J \gtrsim 3.95$, in close agreement with Ref. 11 .

Let us now turn to the spin-wave operators $S_{q}^{+}$and $S_{q}^{z}$, in order to apply the arguments developed in the previous section and assess the nature of the transition. First of all, it should be noticed that, in the $X Y$ model, $S_{q}^{z}$ acts on the ground state by creating a standard spin-wave excitation. This can be easily seen by considering the Holstein-Primakoff representation at the leading order in 


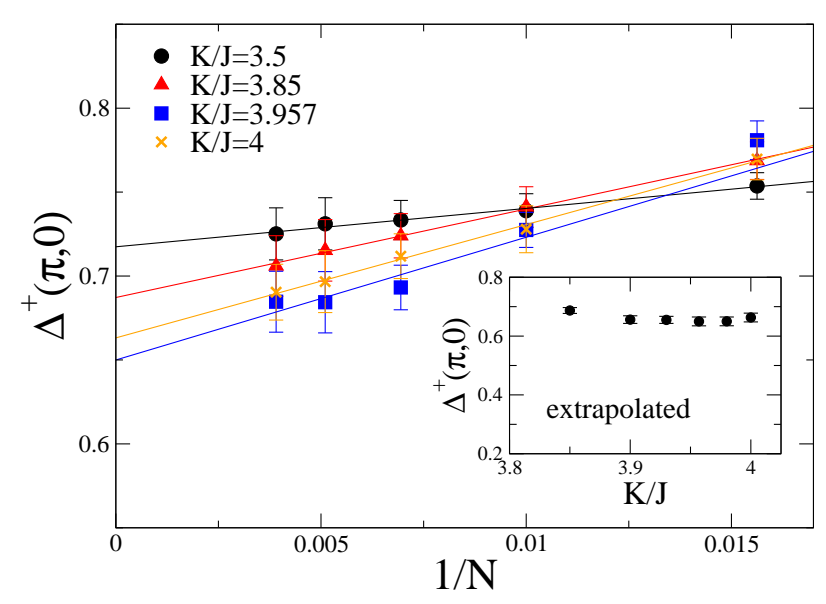

FIG. 4: (Color online) The gap $\Delta^{+}(\pi, 0)$ [in unit of $(K+$ $J)$ ] calculated by GFMC as a function of the size $N$ of the cluster for different values of $K / J$. The lines are linear fits of the points. Inset: the thermodynamic value of the gap as a function of $K / J$.

the $1 / S$ expansion:

$$
\begin{aligned}
S_{j}^{x} & =S-a_{j}^{\dagger} a_{j} \\
S_{j}^{y} & =\sqrt{\frac{S}{2}}\left(a_{j}^{\dagger}+a_{j}\right) \\
S_{j}^{z} & =-i \sqrt{\frac{S}{2}}\left(a_{j}^{\dagger}-a_{j}\right) .
\end{aligned}
$$

After standard calculations, we arrive to the result that

$$
\begin{aligned}
& S_{q}^{y}|0\rangle \sim \alpha_{q}^{\dagger}|0\rangle \\
& S_{q}^{z}|0\rangle \sim \alpha_{q}^{\dagger}|0\rangle,
\end{aligned}
$$

where $|0\rangle$ is the spin-wave ground state and $\alpha_{q}^{\dagger}$ is the creation operator of the elementary excitation, defined by the Bogoliubov transformation. Therefore, the states of the gapless branch have non-zero overlap with the (normalized) states generated either by $S_{q}^{y}$ or by $S_{q}^{z}$, allowing us to assess directly the spin-wave spectrum. As shown in Fig (3), for small values of the ring-exchange coupling, the excitation spectrum remains gapless for $|q| \rightarrow 0$ and the opening of a gap, for $K / J \sim 3.95$, signals the transition to the disordered phase. Moreover, in the ordered phase, the spectrum is linear for small momenta, i.e., $\Delta^{z}(q) \sim c|q|$ (see Fig. 3), the constant $c$ defining the spin-wave velocity. From our numerical results, it comes out that, as assumed in semi-classical approaches, the spin velocity $c$ is not strongly renormalized by frustration and remains finite up to the transition.

By using the same spin-wave operators $S_{q}^{+}$and $S_{q}^{z}$ at $q=(\pi, 0)$ and $q=(0, \pi)$, we can assess the nature of the transition between the ferromagnet and the dimer state. In particular, according to Eq. (12), a continuous phase transition implies a vanishing $\Delta^{+}(q)$ for $q=(\pi, 0)$ and $q=(0, \pi)$. In Fig. (4), we report the results for the gap $\Delta^{+}(\pi, 0)$ for different ratios $K / J$ and various sizes of the

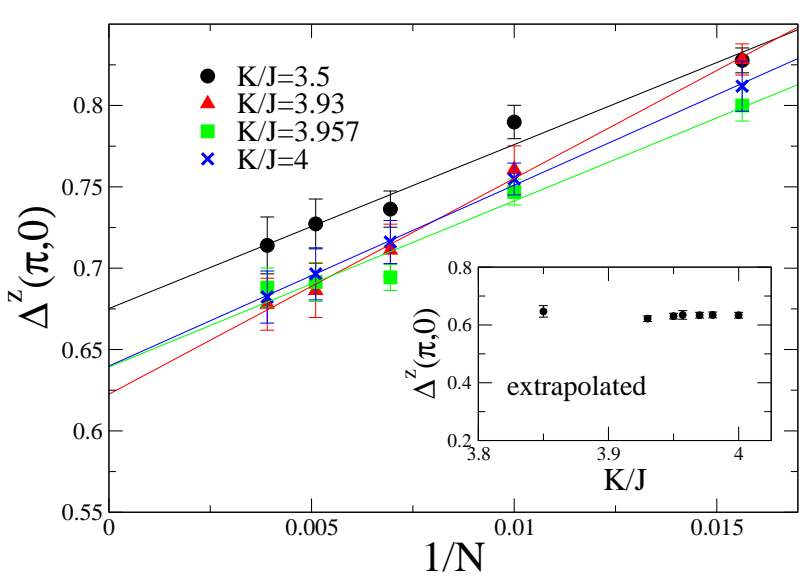

FIG. 5: (Color online) The gap $\Delta^{z}(\pi, 0)$ [in unit of $(K+$ $J)$ ] calculated by GFMC as a function of the size $N$ of the cluster for different values of $K / J$. The lines are linear fits of the points. Inset: the thermodynamic value of the gap as a function of $K / J$.

cluster. From our results, it turns out rather clearly that $\Delta^{+}(\pi, 0)$ remains finite in the thermodynamic limit for all the values of the ring-exchange couplings, also very close to the transition point. Of course, we cannot rule out the existence of a very tiny region, near to the transition point, in which this gap would drop down to zero. Another possibility for missing the vanishing behavior of the gap could be due to an anomalous size scaling, that changes its behavior beyond a characteristic length scale $L_{c}$ much bigger than the values of $L$ considered here. However, from our GFMC calculations, we do not have any sizable sign that could indicate the existence of gapless excitation at $q=(\pi, 0)$ or $q=(0, \pi)$, and even at the transition point $K_{c} / J \sim 3.957$ the extrapolated value of $\Delta^{+}(\pi, 0)$ remains clearly finite. Since a clear dimer order appears as soon as we enter the disordered region, 11 we can safely rule out the possibility to have a spin-liquid phase in between the ferromagnetic and the dimerized phases. Therefore, our results on $\Delta^{+}(\pi, 0)$ strongly suggest that a first-order transition is the more likely scenario in this $\mathrm{O}(2)$ model with ring-exchange interactions. Similarly, also the gap $\Delta^{z}(q)$ at $q=(\pi, 0)$ and $q=(0, \pi)$ does not show any evidence of softening and remains finite at the transition, see Fig. [5]

\section{CONCLUSIONS}

In conclusion, we investigated the excitation spectrum of a spin model close to the transition from a magnetic phase to a dimerized one, which is particularly important in view of the theoretical predictions of Senthil and coworkers. In particular, we considered an $X Y$ model in presence of a frustrating ring-exchange interaction, a model that certainly leads to a magnetic-dimer transition, as recently shown by Sandvik and collaborators 11,13

The central part of our work is the derivation of a gen- 
eral constraint for the excitation spectrum for a broad class of short-range spin Hamiltonians: whenever the transition is second-order a new branch of gapless excitations must exists, besides the ones expected from standard semi-classical approaches. In particular, on the square lattice with a typical four-fold dimer ground state, these modes are spin-wave-like excitations at momenta $q=(0, \pi)$ and $q=(\pi, 0)$. On the contrary, by using a numerically exact technique, we did not find any evidence of these gapless modes. Therefore, our results rule out both conventional and unconventional continuous transition, implying that a first-order transition represents the most likely scenario, in agreement with recent calculations $\frac{15}{15}$ Although we cannot exclude anomalous finite-size effects, that should be particularly important for correlation functions (see Ref. 11), we expect that the excitation spectrum does not suffer from these problems, depending only on total energy differences.

We acknowledge the constant and fruitful interaction with A. Parola, A. Sandvik, and R. Melko. We tank especially L. Capriotti who was involved in the early stage of this project. F.B. thanks F. Alet for very useful discussions. This research has been supported by PRINCOFIN 2004 and INFM. F.B. acknowledges the warm hospitality in the University of Toulouse and its partial support.
1 For a recent review, see for instance, Frustrated Spin systems, Edited by H.T. Diep (World Scientific, Singapore, 2003).

2 P. Fazekas and P.W. Anderson, Philos. Mag. 30, 423 (1974).

3 R. Coldea, D.A. Tennant, A.M. Tsvelik, and Z. Tylczynski, Phys. Rev. Lett. 86, 1335 (2001); R. Coldea, D.A. Tennant, and Z. Tylczynski, Phys. Rev. B 68, 134424 (2003).

4 Y. Shimizu, K. Miyagawa, K. Kanoda, M. Maesato, and G. Saito, Phys. Rev. Lett. 91, 107001 (2003).

5 A.P. Ramirez, G.P. Espinosa, and A.S. Cooper, Phys. Rev. Lett. 64, 2070 (1990).

6 See e.g. the review on $\mathrm{CuGeO}_{3}$ by J.-P. Boucher and L.-P. Regnault, J. Physique 6, 1939 (1996).

7 X.-G. Wen, Phys. Rev. B 65, 165113 (2002).

8 M.B. Hastings, Phys. Rev. B 69, 104431 (2004).

9 T. Senthil, A. Vishwanath, L. Balents, S. Sachdev, and M.P.A. Fisher, Science 303, 1490 (2004).

10 T. Senthil, L. Balents, S. Sachdev, A. Vishwanath, and M.P.A. Fisher, Phys. Rev. B 70, 144407 (2004).

11 A.W. Sandvik, S. Daul, R.R.P. Singh, and D.J. Scalapino, Phys. Rev. Lett. 89, 247201 (2002).

12 A similar model with a ring-exchange term on the Kagome lattice in the Ising-axis limit has been predicted to exhibit a non-trivial quantum phase transition from a magnetically ordered to a spin-liquid phase. D.N. Sheng and L. Balents, Phys. Rev. Lett. 94, 146805 (2005).

13 R.G. Melko, A.W. Sandvik, and D.J. Scalapino, Phys. Rev.
B 69, 100408 (2004).

14 We thanks R.G. Melko for providing us the best estimate of the transition point.

15 From recent numerical calculations, it seems that, from the disordered region, the dimer order parameter vanishes at the transition point, while, from the ordered phase, the spin stiffness shows a small jump. R. Melko and A.W. Sandvik, private communication.

16 M. Calandra Buonaura and S. Sorella, Phys. Rev. B 57, 11446 (1998).

17 R.P. Feynman and M. Cohen, Phys. Rev. 102, 1189 (1956).

18 M. Kamal and G. Murthy, Phys. Rev. Lett. 71, 1991 (1993).

19 O.I. Motrunich and A. Vishwanath, Phys. Rev. B 70, 075104 (2004).

20 For a review on the semi-classical approaches and their relation with field-theory methods, see for instance, J. ZinnJustin, Quantum Field Theory and Critical Phenomena (Clarendon Press, Oxford, 1989).

21 L. Capriotti and F. Becca, Phys. Rev. B 65, 092406 (2002).

${ }^{22}$ F. Franjic and S. Sorella, Prog. Teor. Phys. 97, 399 (1997)

23 S. Sorella, Phys. Rev. B 64, 024512 (2001).

24 S. Yunoki and S. Sorella, Phys. Rev. Lett. 92, 157003 (2004).

25 In this respect, more sophisticated techniques are possible, like for instance the maximum entropy method, that, however, do not change the estimate of the lowest gap excitation. 